\title{
Potency of bisresorcinol from Heliciopsis terminalis on skin aging: in vitro bioactivities and molecular interactions
}

\author{
Charinrat Saechan ${ }^{1}$, Uyen Hoang Nguyen ${ }^{2}$, Zhichao Wang ${ }^{2}$, Sachiko Sugimoto ${ }^{2}$, Yoshi Yamano ${ }^{2}$, Katsuyoshi \\ Matsunami $^{2}$, Hideaki Otsuka ${ }^{3}$, Phan Minh Giang ${ }^{4}$, Pham Hung Viet ${ }^{5}$, Varomyalin Tipmanee ${ }^{\text {Corresp., } 6}$, Jasadee \\ Kaewsrichan ${ }^{\text {Corresp. } 1}$ \\ ${ }^{1}$ Department of Pharmaceutical Chemistry and Drug Delivery System Excellence Center, Faculty of Pharmaceutical Sciences, Prince of Songkla \\ University, Songkhla, Thailand \\ 2 Graduate School of Biomedical and Health Sciences, Hiroshima University, Hiroshima, Japan \\ 3 Faculty of Pharmacy, Yasuda Women's University, Hiroshima, Japan \\ 4 Faculty of Chemistry, VNU University of Science,, Vietnam National University, Hanoi, Vietnam \\ 5 Research Center for Environmental Technology and Sustainable Development, VNU University of Science, Vietnam National University, Hanoi, Vietnam \\ 6 Department of Biomedical Sciences and Biomedical Engineering, Faculty of Medicine, Prince of Songkla University, Songkhla, Thailand \\ Corresponding Authors: Varomyalin Tipmanee, Jasadee Kaewsrichan \\ Email address: tvaromya@medicine.psu.ac.th, jasadee.k@psu.ac.th
}

Background: A bisresorcinol was isolated as the main constituent of Heliciopsis terminalis's trunk (Proteaceae). Recently, resorcinol is applied as an active whitening agent in various cosmetic products. Because of the structural mimic to resorcinol, benefits of the bisresorcinol as an aging-enzyme antagonist were demonstrated in this study.

Methods: The bisresorcinol was purified from the crude ethanolic extract of $H$. terminalis's trunk by solvent extraction and preparative chromatography, respectively. Inhibitory activity on collagenase, elastase, and tyrosinase of the compound was investigated by using a different spectroscopic technique. Molecular docking was carried out to predict possible interactions of the substance around the enzyme active sites.

Results: The $\mathrm{IC}_{50}$ values on collagenase of the bisresorcinol and caffeic acid were $156.7 \pm 0.7$ and $308.9 \pm 1.6 \mu \mathrm{mole} \mathrm{L}^{-1}$, respectively. For elastase activity, the $\mathrm{IC}_{50}$ of $33.2 \pm 0.5$ and $34.3 \pm 0.3 \mu \mathrm{mole} \mathrm{L}^{-1}$ was respectively determined for the bisresorcinol and ursolic acid. The bisresorcinol was inhibitory to tyrosinase by exhibiting the $\mathrm{IC}_{50}$ of $22.8 \mu \mathrm{mole} \mathrm{L}^{-1}$, and that of $78.4 \mu \mathrm{mole}^{-1}$ was present for $\beta$-arbutin. The bisresorcinol bound to collagenase, elastase, and tyrosinase with the respective binding energies of $-5.89,-5.69$, and $-6.57 \mathrm{kcal} \mathrm{mol}^{-1}$. These binding energies were in the same ranges of tested inhibitors. The aromatic phenol groups in the structure were responsible for principle as well as supporting binding interactions with enzymes. Hydrogen binding due to hydroxyl groups and $\pi$-related attractive forces from an aromatic ring(s) provided binding versatility to bisresorcinol.

Conclusion: The bisresorcinol purified from $\mathrm{H}$. terminalis might be useful for inclusion in cosmetic products as an aging-enzyme antagonist. 
1 Potency of bisresorcinol from Heliciopsis terminalis on skin aging: in vitro bioactivities and

2 molecular interactions

3 Charinrat Saechan ${ }^{1}$, Uyen Hoang Nguyen ${ }^{2}$, Zhichao Wang ${ }^{2}$, Sachiko Sugimoto², Yoshi

4 Yamano $^{2}$, Katsuyoshi Matsunami ${ }^{2}$, Hideaki Otsuka ${ }^{3}$, Phan Minh Giang ${ }^{4}$, Pham Hung Viet ${ }^{5}$,

5 Varomyalin Tipmanee ${ }^{6}$, and Jasadee Kaewsrichan ${ }^{1}$

$6{ }^{1}$ Department of Pharmaceutical Chemistry and Drug Delivery System Excellence Center, Faculty

7 of Pharmaceutical Sciences, Prince of Songkla University, Hat-Yai, Songkhla, Thailand

$8{ }^{2}$ Graduate School of Biomedical and Health Sciences, Hiroshima University, 1-2-3 Kasumi,

9 Minami-ku, Hiroshima, Japan

10 35aculty of Pharmacy, Yasuda Women's University, 6-13-1 Yasuhigashi, Asaminami-ku,

11 Hiroshima, Japan

$12{ }^{4}$ Faculty of Chemistry, VNU University of Science, Vietnam National University; 19 Le Thanh

13 Tong, Hanoi, Vietnam

$14{ }^{5}$ Research Center for Environmental Technology and Sustainable Development, VNU University

15 of Science, Vietnam National University, Hanoi, 334 Nguyen Trai Street, Hanoi, Vietnam

$16{ }^{6}$ Department of Biomedical Sciences and Biomedical Engineering, Faculty of Medicine, Prince

17 of Songkla University, Hat-Yai, Songkhla, Thailand

19 Corresponding Authors:

20 Jasadee Kaewsrichan, Department of Pharmaceutical Chemistry, Faculty of Pharmaceutical

21 Sciences, Prince of Songkla University, Hat-Yai, Songkhla, 90112, Thailand

22 E-mail address: jasadee.k@psu.ac.th 
23 Varomyalin Tipmanee, Department of Biomedical Sciences and Biomedical Engineering,

24 Faculty of Medicine, Prince of Songkla University, Hat-Yai, Songkhla, 90112, Thailand

25 Email address: tvaromya@medicine.psu.ac.th

\section{Abstract}

27 Background: A bisresorcinol was isolated as the main constituent of Heliciopsis terminalis's

28 trunk (Proteaceae). Recently, resorcinol is applied as an active whitening agent in various

29 cosmetic products. Because of the structural mimic to resorcinol, benefits of the bisresorcinol as

30 an aging-enzyme antagonist were demonstrated in this study.

31 Methods: The bisresorcinol was purified from the crude ethanolic extract of $H$. terminalis's

32 trunk by solvent extraction and preparative chromatography, respectively. Inhibitory activity on

33 collagenase, elastase, and tyrosinase of the compound was investigated by using a different

34 spectroscopic technique. Molecular docking was carried out to predict possible interactions of

35 the substance around the enzyme active sites.

36 Results: $\mathrm{The} \mathrm{IC}_{50}$ values on collagenase of the bisresorcinol and caffeic acid were $156.7 \pm 2.3$ and

$37308.9 \pm 13.7 \mu \mathrm{mol} \mathrm{L}-1$, respectively. For elastase activity, the $\mathrm{IC}_{50}$ of $33.2 \pm 1.1$ and $34.3 \pm 0.6 \mu \mathrm{mol}$

$38 \mathrm{~L}^{-1}$ was respectively determined for the bisresorcinol and ursolic acid.

39 The bisresorcinol was inhibitory to tyrosinase by exhibiting the $\mathrm{IC}_{50}$ of $22.6 \mu \mathrm{mol} \mathrm{L}-1$, and that

40 of $78.5 \mu \mathrm{mol} \mathrm{L}^{-1}$ was present for $\beta$-arbutin. The bisresorcinol bound to collagenase, elastase, and

41 tyrosinase with the respective binding energies of $-5.89,-5.69$, and $-6.57 \mathrm{kcal} \mathrm{mol}^{-1}$. These

42 binding energies were in the same ranges of tested inhibitors. The aromatic phenol groups in the

43 structure were responsible for principle as well as supporting binding interactions with enzymes.

44 Hydrogen binding due to hydroxyl groups and $\pi$-related attractive forces from an aromatic

45 ring(s) provided binding versatility to bisresorcinol. 
46 Conclusion: The bisresorcinol purified from $H$. terminalis might be useful for inclusion in

47 cosmetic products as an aging-enzyme antagonist.

\section{Introduction}

49 A bisresorcinol, (8'Z)-3,5-dihydroxy-1-[16'-(3",5"-dihydroxyphenyl)-8'-hexadecen-1'-yl]benzene, 50 has been isolated as an abundant constituent from the trunk of Heliciopsis terminalis (Kurz)

51 Sleumer, which is a plant of Proteaceae family and has been used by ethnic people in Vietnam 52 for hepatoprotective effect. To date, medical benefits of this compound as an agent for anti53 inflammation, inhibition of malondialdehyde (MDA) production, and hepatoprotection, have 54 been clarified in vitro and in vivo (Giang et al., 2019). It is apparent in that a molecule of the 55 bisresorcinol contains two resorcinol molecules (EC No 203-585-2), bonded together with meta$56 \mathrm{C}_{7} \mathrm{H}_{14} \mathrm{C}=\mathrm{CC}_{7} \mathrm{H}_{14}$ linkage (Fig. 1a). Resorcinol is recently applied in a form of facial concentrated 57 serum for white therapy. Based on the Scientific Committee on Consumer Safety (SCCS 58 1270/09, European Commission, 2010), resorcinol has been regulated as an oxidant in products 59 intended for coloring eyelashes at concentration ranges up to $1.25 \%$, or in hair lotions and 60 shampoos at concentration ranges up to $0.5 \%$. Cosmetical applications of resorcinol are thus 61 diverse. For whitening agents, inhibition of melanin synthesis by melanocytes that reside in the 62 basal cell layer of epidermis has been assumed. Tyrosinase (EC 1.14.18.1) is a key enzyme for 63 catalyzing melanogenesis in which L-tyrosine is used as the substrate. Although melanin helps 64 protect skin form damage of UV rays, excess melanin production can cause hyperpigmentation, 65 freckle, and age spot. In addition to resorcinol, various synthetic and natural compounds with 66 distinct chemical structures have been demonstrated to exhibit anti-tyrosinase activity (Dobos et 67 al., 2015). 

time by reactive oxygen species (ROS) and lipid peroxides that are internally produced after UV exposure. Secondary products from lipid peroxide metabolisms can damage elastin and collagen fibers of extracellular matrices (ECMs). Therefore, exposure to UV rays can result in periodically remodeled to maintain normal tissue structures in accompany with that expired

74 tissue proteins are properly degraded by matrix metalloproteinases, such as collagenases, and serine proteases like elastases (Thring, Hili \& Naughton, 2009). In addition, both quantities and activities of aging enzymes have been increased by oxidative stress, leading to extensive ECMs

77 degradation and progressive aging (Sherratt et al., 2019). Therefore, compounds that can form 78 complexes with metal ions are anticipated to be inhibitory to collagenases, elastases, and 79 tyrosinase accordingly (Selvaraj et al., 2014). This project aims to determine biological effects of 80 the bisresorcinol on tyrosinase, collagenase, and elastase in vitro, and docking technique was carried out to clarify their interactions at molecular levels. It is hopeful to delay the aging process

82 and improve skin appearance by the bisresorcinol.

\section{Materials \& Methods}

\section{Chemicals and Instruments}

86 All the chemicals used in this study were of analytical grade. Organic solvents, including 1-

87 butanol, acetone, chloroform (also for NMR), and ethyl acetate, were purchased from KANTO

88 CHEMICAL CO., INC., Japan. Chemicals, such as hydrochloric acid, $\mathrm{K}_{2} \mathrm{HPO}_{4}, \mathrm{KH}_{2} \mathrm{PO}_{4}$, L89 tyrosine, silica gel, octadecyl silica gel, and tris (hydroxymethyl) aminomethane, were bought 90 from NACALAI TESQUE, INC., Japan. Standard substances, including $\beta$-arbutin, caffeic acid, 
91 and ursolic acid, and tricine were obtained from Tokyo Chemical Industry, Japan. Enzymes, such

92 as collagenase, elastase, and tyrosinase, including N-succinyl-Ala-Ala-Ala-p-nitroanilide

93 (SANA) and dimethyl sulfoxide (DMSO), were acquired from Sigma-Aldrich, Germany. DMSO

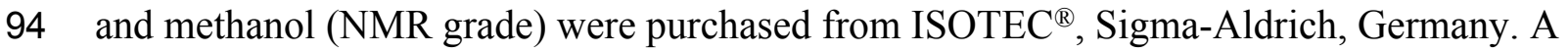

95 peptide: MOCAc-PRO-Leu-Gly-Leu-A 2 pr(Dnp)-Ala-Arg-NH $\mathrm{N}_{2}$, was ordered from Peptide

96 Institute, Inc., Japan. Instruments used included HPLC (SCL-10A sp/RID-6A/c-R3A,

97 SHIMADZU), Fluorescence microplate reader (Enspire 2300 Multimode reader, PerkinElmer),

98 HR-ESI-MS (LTQ orbitrap XL, Thermo Fisher Scientific), Infrared Spectrophotometer (FT-720,

99 HORIBA), Nuclear Magnetic Resonance Spectrometer (ECA-500/600, JEOL), and UV-Vis

100 microplate reader (Multiskan Go, Thermo Scientific).

\section{Compound Purification, Identification and Ratification}

102 The crude extract in ethanol of $H$. terminalis's trunk was generously obtained from Professor

103 P.M. Giang, Faculty of Chemistry, Vietnam National University, Hanoi, Vietnam with voucher

104 specimen number of HNIP-18473. Then, it was partitioned in methanol/n-hexane by 3 times

105 repeated. The methanol layer was evaporated to nearly dry and subsequently partitioned in ethyl

106 acetate/1-butane. The ethyl acetate layer was vaporized until its volume was reduced by $80 \%$.

107 The resulting viscous liquid was then purified on silica gel column using chloroform/methanol

108 gradient elution as follows: $100 \%$ chloroform, 30:1, 20:1, 10:1, 7:1, 5:1, 3:1, 3:2, and 2:1 of

109 chloroform/methanol, and 100\% methanol, respectively. The eluent from the 5:1 mixed solvent

110 was collected and evaporated to one fourths volume, followed by the separation on the ODS

111 column using gradient elution of methanol and acetone. The desired compound, i.e.,

112 bisresorcinol (Fig. 1), was obtained from eluents containing 70\% acetone and confirmed by MS, 
113 NMR and IR spectroscopies in regard to the instrumental library data. Thin layer

114 chromatography was used for estimation of its purity.

115 Biological Activity Assays

116 In vitro experiments that consisted of enzyme-inhibitory assays on collagenase, elastase and 117 tyrosinase for the bisresorcinol and known inhibitors were carried out as follows.

\section{Collagenase inhibition}

119 The recent anti-collagenase assay was modified from that previously described by Widyowati et 120 al. (2016). In brief, $50 \mathrm{mmol} \mathrm{L}^{-1}$ tricine buffer $\mathrm{pH} 7.5$ supplemented with $400 \mathrm{mmol} \mathrm{L}^{-1} \mathrm{NaCl}$

121 and $10 \mathrm{mmol} \mathrm{L}^{-1} \mathrm{CaCl}_{2}$ was used as the buffering diluent. Collagenase from Clostridium

122 histolyticum (EC.3.4.24.3) was dissolved in the buffer to a concentration of $1 \mathrm{mg} \mathrm{L} \mathrm{L}^{-1}$. The 123 enzyme substrate, MOCAc-PRO-Leu-Gly-Leu-A 2 pr (Dnp)-Ala-Arg- $\mathrm{NH}_{2}$, was prior dissolved in 124 DMSO and subsequently diluted in the buffer to a concentration of $1 \mathrm{mmol} \mathrm{L}^{-1}$. A sample 125 dissolved in DMSO was diluted to different concentrations by using the buffer. A 2- $\mu$ l sample 126 solution was incubated with $100 \mu 1$ enzyme solution at $37^{\circ} \mathrm{C}$ for $10 \mathrm{~min}$. Then, a $50-\mu 1$ substrate 127 was added and thoroughly mixed. Fluorescence emission (F) at $405 \mathrm{~nm}$ was immediately 128 recorded and continually monitored for 30 min using a wavelength of $320 \mathrm{~nm}$ for excitation.

129 Caffeic acid was used as a position control (Fig. 1). Negative control was performed with water. 130 The percent inhibition (\%) was calculated from the equation below:

$$
\% \text { Inhibition }=\left\{1-\left(\mathrm{F}_{\text {sam }, 30}-\mathrm{F}_{\text {sam }, 0}\right) \div\left(\mathrm{F}_{\text {cont }, 30}-\mathrm{F}_{\text {cont }, 0}\right)\right\} \times 100
$$

\section{Elastase Inhibition}

133 The assay according to Abhijit \& Manjushree (2010) was applied with some modifications.

134 Briefly, the buffer system was $0.2 \mathrm{mM}$ Tris-HCl buffer $(\mathrm{pH} 8.0)$. A solution of $1 \mu \mathrm{g} \mathrm{mL}^{-1}$ 
135 porcine pancreatic elastase (EC.3.4.21.36) was prepared in sterile water. The enzyme substrate,

136 N-Succinyl-Ala-Ala-Ala-p-nitroanilide (SANA), was dissolved in the buffer to a concentration

137 of $80 \mathrm{mmol} \mathrm{L}^{-1}$. A sample dissolved in DMSO was diluted to various concentrations by using

138 the buffer. A $100-\mu 1$ sample solution was mixed with $50 \mu 1$ enzyme solution and incubated for 15

$139 \mathrm{~min}$. After that $50 \mu \mathrm{l}$ of the enzyme substrate solution was added and thoroughly mixed. The

$140 \mathrm{OD}_{410}$ was measured immediately $(0 \mathrm{~min})$ and after incubation overnight $(\mathrm{o} / \mathrm{n})$ at $37^{\circ} \mathrm{C}$ by using a

141 microplate reader. Ursolic acid was used as a positive control (Fig. 1), and water was used as a

142 negative control. The percentage inhibition (\%) was calculated by the following equation:

$$
\% \text { Inhibition }=\left\{1-\left[\left(\mathrm{A}_{\mathrm{sam}, \mathrm{o} / \mathrm{n}}-\mathrm{A}_{\mathrm{sam}, 0}\right) \div\left(\mathrm{A}_{\text {cont }, \mathrm{o} / \mathrm{n}}-\mathrm{A}_{\text {cont }, 0}\right)\right]\right\} \times 100 .
$$

\section{Tyrosinase Inhibition}

145 The used tyrosinase inhibitory assay was adopted from the previously described method

146 (Jiratchayamaethasakul et al., 2020). In brief, the assay was carried out in $0.05 \mathrm{~mol} \mathrm{~L}^{-1}$

147 phosphate buffer $\mathrm{pH}$ 6.8. Mushroom tyrosinase (EC.1.14.18.1) was dissolved in the buffer to a

148 concentration of 100 units $\mathrm{mL}^{-1}$. L-tyrosine, an enzyme substrate, was prepared to a

149 concentration of $0.25 \mathrm{mg} \mathrm{mL}^{-1}$ in the buffer. A test compound dissolved in DMSO was diluted to

150 concentrations ranging between 0.625 and $10 \mathrm{mg} \mathrm{mL}^{-1}$. Into a well of 96-well plates, $10 \mu 1$

151 sample and $40 \mu 1 \mathrm{~L}$-tyrosine solution were mixed and incubated for $10 \mathrm{~min}$ at room temperature.

152 After that $50 \mu 1$ enzyme solution was inoculated and mixed thoroughly. The reaction was

153 stopped afterward by incubation on ice for $1 \mathrm{~min}$. The $\mathrm{OD}_{475}$ was measured using a microplate

154 reader. $\beta$-Arbutin and water were used as a positive control (Fig. 1) and a negative counterpart,

155 respectively. The percentage inhibition (\%) was calculated by the equation as follows:

$$
\% \text { Inhibition }=\left\{1-\left(\mathrm{A}_{\mathrm{sam}, 10}-\mathrm{A}_{\text {sam }, 0}\right) \div\left(\mathrm{A}_{\text {cont }, 10}-\mathrm{A}_{\text {cont }, 0}\right)\right\} \times 100 .
$$




\section{Molecular Docking Study}

\section{Preparation of Ligands and Receptors}

159 Three-dimensional (3D) structures of bisresorcinol (CID 8917124) and reference compounds, 160 including substrates and inhibitors, were downloaded from PubChem database for molecular 161 docking study. A ligand structure was prepared in a Protein Data Bank (PDB) format file using 162 Online SMILES Translator and Structure File Generator (https://cactus.nci.nih.gov/translate/).

163 The crystal structures of enzymes, such as collagenase (PDB ID 2Y6I), elastase (PDB ID 164 1BRU), and tyrosinase (PDB ID 2Y9X) were obtained from RCSB protein database 165 (http://www.rcsb.org). The water and the attached molecules were dissected from all selected 166 protein structures. Meanwhile, polar hydrogen atoms were added in the crystallized protein 167 structures by AutoDockTools 1.5.6. Files of target proteins and other used compounds were 168 saved in PDBQT format before performing the molecular docking.

\section{Selection of Active Site Residues and Molecular Docking}

170 Grid and docking protocols of the active site predictions were prepared using AutoDockTools

171 1.5.6. Grid sites were set spacing of $0.375 \AA$. The $x-y-z$ dimensions were set to be $126-135-160$

$172 \AA^{3}$ for collagenase, $130-120-126 \AA^{3}$ for elastase, and $80-80-80 \AA^{3}$ for tyrosinase. Grid box 173 centers (with offset values in AutoDockTools) were 31.869 (5.000), -19.41 (-25.000), and 17.815

174 for collagenase, $30.048(-0.972), 51.253(-2.722), 17.6$ for elastase, and $-8.407(-1.000),-23.795$

$175(-0.250),-36.019(-3.500)$ for tyrosinase, respectively. The protein structure was used as a rigid 176 entity while the ligand compound was set as a flexible molecule. Docking study was performed 177 using the Lamarckian genetic algorithm (GA) implemented by AutoDock4 version 4.2. The 178 number of GA runs was 50 with a popular size of 200 . The binding energy $\left(\Delta \mathrm{G}_{\mathrm{bind}}\right)$ was analyzed 179 by ADT. Interaction(s) was visualized using BIOVIA Discovery Studio (BIOVIA, 2020). 
180 Molecular interaction between the compound and a protein receptor was analyzed and visualized 181 using BIOVIA Discovery Studio software (BIOVIA, 2020). The structure of protein binding site 182 compound was visualized using Visual Molecular Dynamics (VMD) package (Humphrey, Dalke $183 \&$ Schulten, 1996).

184 Statistical Analysis

185 For in vitro study, each experiment was done in triplicate. Data were expressed as means \pm 186 standard error of the mean (SEM). The Student's t-test was used to evaluate differences between 187 results, and $p$-values of $<0.01$ were indicated to be significantly different. The performance 188 regarding statistics was run on Libre Office Calc 5 in Ubuntu 16.04.6 LTS software. 189

\section{Results}

191 A major constituent was successfully purified from $H$. terminalis's trunk by using solvent 192 extraction and reverse phase HPLC techniques, respectively. The acquired ${ }^{1} \mathrm{H}-\mathrm{NMR}$ and ${ }^{13} \mathrm{C}$ 193 NMR spectra were compared to those of reference substances through the SciFinder 194 (https://scifinder.cas.org/) and found to correspond with (8'Z)-1,3-dihydroxy-5-[16'-(3",5"195 dihyroxyphenyl)-8'-hexadecen-1'-yl] benzene (Chaturvedula et al., 2002). It was classified as one 196 of bisresorcinols. This recent bisresorcinol had the molecular formula of $\mathrm{C}_{28} \mathrm{H}_{40} \mathrm{O}_{4}$ with the 197 molecular weight of 463.2819 Dalton. The extraction yield was calculated to be $0.086 \%$, based 198 on the trunk' dried weight. Its purity was more than $98 \%$, concerning the ${ }^{1} \mathrm{H}$ and ${ }^{13} \mathrm{C}$-NMR 199 spectra acquired (see Figure S1 and S2). 


\section{In vitro bioactivity analysis}

201 Antagonistic effects of the bisresorcinol on aging enzymes, such as collagenase, elastase, and

202 tyrosinase were determined in vitro by using a distinct spectroscopic method. Results were

203 summarized in Table 1 and Fig. 2. For collagenase inhibition, test samples in a range of 50-550

$204 \mu \mathrm{mol} \mathrm{L}{ }^{-1}$ were prepared using MOCAc-PRO-Leu-Gly-Leu-A 2 pr (Dnp)-Ala-Arg-NH ${ }_{2}$ as the

205 enzyme substrate and caffeic acid as an enzyme inhibitor. It was indicated that the bisresorcinol

206 at a concentration of $156.7 \mu \mathrm{mol} \mathrm{L}-1$ decreased the enzyme activity by $50 \%\left(\mathrm{IC}_{50}\right)$. Instead, the

$207 \mathrm{IC}_{50}$ of caffeic acid was $308.9 \pm 13.7 \mu \mathrm{mol} \mathrm{L}{ }^{-1}$. Thus, anti-collagenase activity of the bisresorcinol

208 was significantly stronger than caffeic acid.

209

Inhibition of elastase enzyme was determined at a concentration range between 10 and

$210100 \mu \mathrm{mol} \mathrm{L}-1$. The residual enzyme activity was spectroscopically measured at $410 \mathrm{~nm}$ in

211 response to the amount of $p$-nitroaniline released from the substrate SANA after cleavage. The

212 bisresorcinol strongly inhibited elastase activity in a concentration-dependent manner with the

213 maximum inhibition of $100 \%$ at $50 \mu \mathrm{mol} \mathrm{L}-1$. However, the inhibition decreased rapidly at

214 concentrations less than $30 \mu \mathrm{mol} \mathrm{L}{ }^{-1}$. Notably, the dose-response curve of ursolic acid was less

215 steep than that of the bisresorcinol by reaching $80 \%$ inhibition at $100 \mu \mathrm{mol} \mathrm{L}^{-1}$. The $\mathrm{IC}_{50}$ values

216 of the bisresorcinol and ursolic acid were respectively 33.2 and $34.3 \mu \mathrm{mol} \mathrm{L}^{-1}$, which were not

217 significantly different.

218 In analysis of tyrosinase inhibition, samples were diluted to a concentration range of 10-

$219100 \mu \mathrm{mol} \mathrm{L}^{-1}$. L-tyrosine was used as a substrate of mushroom tyrosinase and $\beta$-arbutin was a

220 known inhibitor. The bisresorcinol showed the $\mathrm{IC}_{50}$ of $22.6 \mu \mathrm{mol} \mathrm{L}{ }^{-1}$. The $\mathrm{IC}_{50}$ of $\beta$-arbutin was

$22178.5 \mu \mathrm{mol} \mathrm{L}{ }^{-1}$. Our test compound showed $100 \%$ inhibition at $50 \mu \mathrm{mol} \mathrm{L}^{-1}$. In contrast, the

222 enzyme inhibition of $\beta$-arbutin higher than $50 \%$ was not determined in the tested range.

PeerJ reviewing PDF | (2020:12:55952:1:1:NEW 30 Apr 2021) 


\section{Molecular interaction analysis}

224 Molecular docking was applied to predict binding sites of test compounds to protein receptors,

225 such as collagenase, elastase and tyrosinase, in comparison with known inhibitors of a

226 corresponding enzyme (supplement information). Consisting with previous publications, the

227 relative binding affinity was evaluated meanwhile binding interactions were illustrated through

228 the best predicted conformation (Teajaroen et al., 2020; Jewboonchu et al., 2020;

229 Tanawatsoontorn et al., 2020; Saeloh et al., 2017).

230 For collagenase enzyme, results of ligand-protein interactions were demonstrated in Fig.

2313 and Table S1 (supplement data). The binding energy of $-5.89 \mathrm{kcal} \mathrm{mol}^{-1}$ to clostridial

232 collagenase (PDB ID 2Y6I) was presented by the bisresorcinol. A range of the binding energy

233 between -3.68 and $-7.90 \mathrm{kcal} \mathrm{mol}^{-1}$ was determined for other known collagenase inhibitors,

234 including caffeic acid.

235 Both the bisresorcinol and caffeic acid shared the collagenase binding sites (Fig. 3A).

236 Four amino acids responsible for caffeic acid attachment included His524, Trp496, His527, and

237 Trp539 (Fig. 3B). His524 and Trp496 interacted caffeic acid by hydrogen bonds through

238 carboxylic and phenolic hydroxyl group(s). His527 and Trp539 bonded to the phenolic ring by

$239 \pi-\pi$ stacking. Other amino acids within the enzyme pocket contributed their binding through Van

240 der Waals force (Fig. 3C). Interactions between the collagenase and the bisresorcinol were

241 shown in the Fig. 3D and E, involving Trp496 and Trp539 amino acids that donated $\pi$-electrons

242 to bond to the phenolic ring of the bisresorcinol. The presence of hydrogen bonds between the

243 hydroxyl groups of the bisresorcinol and amino acids Asp601 and Ser602 was observed.

244 Moreover, the $\mathrm{Zn}$ atom in the enzyme active site might be coordinated to both the bisresorcinol

245 and caffeic acid. 
behaviors to elastase enzyme of the bisresorcinol and other known inhibitors, including ursolic

all test compounds ranged between -5.62 and $-8.94 \mathrm{kcal} \mathrm{mol}^{-1}$ and that of $-5.69 \mathrm{kcal} \mathrm{mol}^{-1}$ was calculated for the bisresorcinol.

There was similarity in binding to the elastase of the bisresorcinol and ursolic acid (Fig. group and Ser96, as well as a carboxylic group and Asn192 (Fig. 4B and C). Interestingly, structural folding of the bisresorcinol was presumed. This might facilitate interactions between phenolic hydroxyl groups of the compound and amino acids like Asn147, Ser190, Phe215, and Ser217 through hydrogen bonding (Fig. 4D and E). mushroom tyrosinase (PDB ID 2Y9X) was applied. Results were displayed in Fig. 5 and Table S3 (supplement information), indicating that the binding energy of the bisresorcinol was -6.57 $\mathrm{kcal} \mathrm{mol}{ }^{-1}$, being in a range of -4.63 to $-8.12 \mathrm{kcal} \mathrm{mol}^{-1}$ for other known inhibitors. In contrast, all known substrates were tightly bound to the tyrosinase according to an enormous decrease of the binding energy to a range from -15.46 and $-23.94 \mathrm{kcal} \mathrm{mol}^{-1}$. the tyrosinase active site likely occurred. However, copper ions $\left(\mathrm{Cu}^{2+}\right)$ were replaced by zinc ions $\left(\mathrm{Zn}^{2+}\right)$ in the AutodockTool, because the force field for $\mathrm{Cu}^{2+}$ is unavailable and $\mathrm{Cu}^{2+}$ and $\mathrm{Zn}^{2+}$ ions are quite identical in charges and sizes (Santos-Martins et al., 2014). For $\beta$-arbutin, one

267 zinc ion might covalently bond to the phenolic hydroxyl group, and another zinc ion interacted 268 with the phenol ring through $\pi$-cation bond with His259 and His263 residues (Fig. $5 \mathrm{~B}$ and C). In 
269 addition, the amino acids, such as Asn260, Ser282, and Val283, are linked to its hydroxyl groups

270 by hydrogen bonding. In view of the bisresorcinol, the phenolic hydroxyl group formed

271 hydrogen bonds with Met280, Ala 246, and Glu239. The $\pi-\pi$ stacked interaction between the

272 phenol ring and His263, and the $\pi$-cation attraction between another phenol ring and Arg321

273 were distinctly observed.

274 In summary, molecular interactions and binding affinity between the bisresorcinol and 275 collagenase, elastase, or tyrosinase were tabulated in Table 2.

276

\section{Discussion}

278 Curcuminoid, a phenolic compound from Curcuma longa L., has been included in various 279 cosmetic products as an antioxidant for anti-aging property (Gopinath \& Karthikeyan, 2018).

280 Since phenolic hydroxy determinants were existent, the bisresorcinol from H. terminalis's trunk 281 was anticipated to harbor anti-aging activity accordingly (Fig. 1). In the recent in vitro inhibitory 282 assays, the bisresorcinol was found to dominantly inhibit elastase and tyrosinase activities rather 283 than collagenase counterpart (Fig. 2). Accordingly, in silico docking experiments were 284 performed to ascertain binding affinity of the bisresorcinol relevant to these aging enzymes.

285 Interactions regarding $\pi$-electrons and hydrogen bonds were assumed to be key determinants for 286 its bindings. Moreover, hydrophobic interactions that occurred between phenolic hydroxyl 287 groups and amino acid residues at/nearby the enzyme active sites might be contributors for loss 288 of enzyme functions, consisting with the previous literatures (Medvidović-Kosanović et al., 289 2010; Pientaweeratch, Panapisal \& Tansirikongkol, 2016). Herein, binding characteristics of the 290 bisresorcinol to each enzyme were distinctly explained based on structural comparison 291 corresponding to a specific inhibitor. Inhibition of clostridial collagenase by caffeic acid, EGCG, 
292 quercetin, and catechin has been documented (Szewczyk et al., 2020; Pluemsamran et al., 2012;

293 Hong et al., 2014). Similar to other known inhibitors, the hydroxy phenol groups of the

294 bisresorcinol were suggested to interact with key amino acids of the collagenase binding site

295 through $\pi-\pi$ interaction and hydrogen bonding. Interestingly, the long flexible structure of

296 bisresorcinol had a capability to fold or elongate. Thus, when elongated, the unbound phenol ring

297 on the other side could bind to amino acid residues at the active site, resulting in increased

298 binding strength. By this way, inhibitory potential of the bisresorcinol on collagenase activity

299 was proposed (Fig. 6).

300 In agreement with a previous study (Huang et al., 2013), binding to elastase enzyme of

301 both the bisresorcinol and known inhibitors, such as procyanidin, quercetin and ursolic acid,

302 were identical, involving hydrogen bond formation. Moreover, interactions between the

303 bisresorcinol and amino acids apart from the enzyme pocket were speculated to mediate the

304 unfolded phenol ring of the substance (Fig. 7).

305 In view of tyrosinase inhibitory activity, inhibitors like kojic acid, rutin, and L-mimosine,

306 have been apparent to competitively bind to tyrosinase enzyme with L-tyrosine, leading to

307 inhibition of melanin synthesis (Channar et al., 2018; Nguyen \& Tawata, 2015; Si et al., 2012).

308 In Fig. 8, the $\pi-\pi$ interactions between any test compound and histidine residues of the copper

309 active site of tyrosinase were suggested. In agreement with a previous research, this particularly

310 provided an explanation for other antagonistic effects on tyrosinase activity of such compounds

311 (Lai et al., 2017). In fact, the phenolic skeleton in structures has been implicated in designing

312 derivatives of indanone (Jung et al., 2019) and thiazolyl resorcinol (Mann et al., 2018),

313 supporting the possibility of using the bisresorcinol as an antagonist of tyrosinase enzyme. 


\section{Conclusion}

316 The bisresorcinol might serve as a competitive inhibitor for collagenase, elastase, and tyrosinase

317 with comparable modes of binding compared to known inhibitors. However, the long and

318 flexible structure of the bisresorcinol was interesting in that additional interactions towards an

319 unbound phenol ring to neighboring amino acids of the enzymes might be apparent. This finding

320 was firstly reported and might give an idea for development of new cosmetic products containing

321 the bisresorcinol for anti-aging and whitening effects. Nevertheless, there were further needs to

322 examine its potency in vivo and by clinical trials.

323

324 Acknowledgements

325 The authors sincerely thank to other research facility providers, such as the Graduate School at 326 Prince of Songkla University; Drug Delivery System Excellence Center, Faculty of

327 Pharmaceutical Sciences, Prince of Songkla University; the Graduate School of Biomedical and

328 Health Sciences (Pharmaceutical Sciences), Hiroshima University, Hiroshima, Japan; and

329 Department of Biomedical Sciences and Biomedical Engineering, Faculty of Medicine, Prince of 330 Songkla University, Thailand.

331

332 References

333 Abhijit S, Manjushree D. 2010. Anti-hyaluronidase, anti-elastase activity of Garcinia indica. 334 International Journal of Botany 6(3):299-303 DOI 10.3923/ijb.2010.299.303. 
335 BIOVIA. 2020. Discovery studio. San Diego: Dassault Systèmes.

336 Channar PA, Saeed A, Larik FA, Batool B, Kalsoom S, Hasan MM, Ashraf Z. 2018.

337 Synthesis of aryl pyrazole via Suzuki coupling reaction, in vitro mushroom tyrosinase

338 enzyme inhibition assay and in silico comparative molecular docking analysis with kojic acid. Bioorganic Chemistry 79:293-300 DOI 10.1016/j.bioorg.2018.04.026.

Chaturvedula VP, Schilling JK, Miller JS, Andriantsiferana R, Rasamison VE, Kingston DG. 2002. New cytotoxic bis 5-alkylresorcinol derivatives from the leaves of Oncostemon bojerianum from the Madagascar rainforest. Journal of natural products 65(11):1627-1632 DOI $10.1021 / \mathrm{np} 0201568$.

Dobos G, Lichterfeld A, Blume-Peytavi U, Kottner J. 2015. Evaluation of skin ageing: a systematic review of clinical scales. British Journal of Dermatology 172(5):1249-1261 DOI 10.1111/bjd.13509.

347

348

349

350

351

352

353

354

355

356

357

European Commission, Scientific Committee on Consumer Safety. 2010. Opinion on resorcinol, SCCS/1270/09. Available at: https://ec.europa.eu/health/scientific_ committees/consumer_safety/docs/sccs_o_015.pdf (accessed 19 February 2021)

Giang PM, Nga NT, Van Trung B, Anh DH, Viet PH. 2019. Evaluation of the antioxidant, hepatoprotective, and anti-inflammatory activities of bisresorcinol isolated from the trunk of Heliciopsis terminalis. Pharmaceutical Chemistry Journal 53(7):628-634 DOI 10.1007/s11094-019-02051-7.

Gopinath H, Karthikeyan K. 2018. Turmeric: a condiment, cosmetic and cure. Indian Journal of Dermatology Venereology and Leprology 84(1):16 DOI 10.4103/ijdvl.IJDVL_1143_16.

Hong YH, Jung EY, Noh DO, Suh HJ. 2014. Physiological effects of formulation containing tannase-converted green tea extract on skin care: physical stability, collagenase, elastase, and 
tyrosinase activities. Integrative medicine research 3(1):25-33 DOI

359 10.1016/j.imr.2013.12.003.

360

361

362

363

364

365 366

367

368

369

370

371

372

373

374

375

376

377

378

Huang Y, Chen L, Feng L, Guo F, Li Y. 2013. Characterization of total phenolic constituents from the stems of Spatholobus suberectus using LC-DAD-MS ${ }^{\mathrm{n}}$ and their inhibitory effect on human neutrophil elastase activity. Molecules 18(7):7549-7556 DOI 10.3390/molecules 18077549.

Humphrey W, Dalke A, Schulten K. 1996. VMD - Visual Molecular Dynamics. Journal of Molecular Graphics 14(1):33-38 DOI 10.1016/0263-7855(96)00018-5.

Jewboonchu J, Saetang J, Saeloh D, Siriyong T, Rungrotmongkol T, Voravuthikunchai SP, Tipmanee V. 2020. Atomistic insight and modeled elucidation of conessine towards Pseudomonas aeruginosa efflux pump. Journal of Biomolecular Structure and Dynamics Oct 7:1-10 DOI 10.1080/07391102.2020.1828169.

\section{Jiratchayamaethasakul C, Ding Y, Hwang O, Im ST, Jang Y, Myung SW, Lee JM, Kim} HS, Ko SC, Lee SH. 2020. In vitro screening of elastase, collagenase, hyaluronidase, and tyrosinase inhibitory and antioxidant activities of 22 halophyte plant extracts for novel cosmeceuticals. Fisheries and Aquatic Sciences 23(1):1-9 DOI 10.1186/s41240-020-001498.

Jung HJ, Noh SG, Park Y, Kang D, Chun P, Chung HY, Moon HR. 2019. In vitro and in silico insights into tyrosinase inhibitors with (E)-benzylidene-1-indanone derivatives. Computational and structural biotechnology journal 17:1255-1264. DOI 10.1016/j.csbj.2019.07.017. 
379 Lai X, Wichers HJ, Soler-Lopez M, Dijkstra BW. 2017. Structure of human tyrosinase related

380 protein 1 reveals a binuclear zinc active site important for melanogenesis. Angewandte

381 Chemie International Edition 56(33):9812-9815 DOI 10.1002/anie.201704616.

382

383

384

385

386

387

388

389

390

391

392

393

394

395

396

397

398

399

400

401

Mann T, Scherner C, Röhm KH, Kolbe L. 2018. Structure-activity relationships of thiazolyl resorcinols, potent and selective inhibitors of human tyrosinase. International journal of molecular sciences 19(3):690 DOI 10.3390/ijms19030690.

Medvidović-Kosanović M, Šeruga M, Jakobek L, Novak I. 2010. Electrochemical and antioxidant properties of (+)-catechin, quercetin and rutin. Croatica chemica acta 83(2):197207

Nguyen BCQ, Tawata S. 2015. Mimosine dipeptide enantiomsers: improved inhibitors against melanogenesis and cyclooxygenase. Molecules 20(8):14334-14347 DOI 10.3390/molecules200814334.

Pientaweeratch S, Panapisal V, Tansirikongkol A. 2016. Antioxidant, anti-collagenase and anti-elastase activities of Phyllanthus emblica, Manilkara zapota and silymarin: an in vitro comparative study for anti-aging applications. Pharmaceutical biology 54(9):1865-1872. DOI 10.3109/13880209.2015.1133658.

Pluemsamran T, Onkoksoong T, Panich U. 2012. Caffeic acid and ferulic acid inhibit UVAinduced matrix metalloproteinase-1 through regulation of antioxidant defense system in keratinocyte HaCaT cells. Photochem. Photobiol 88:961-968 DOI 10.1111/j.17511097.2012.01118.x.

Saeloh D, Wenzel M, Rungrotmongkol, T, Hamoen, LW, Tipmanee V, Voravuthikunchai SP. 2017. Effects of rhodomyrtone on Gram-positive bacterial tubulin homologue FtsZ. PeerJ 5:e2962 DOI doi: 10.7717/peerj.2962. 
402 Santos-Martins D, Forli S, Ramos MJ, Olson AJ. 2014. AutoDock4 ${ }_{Z n}$ : an improved AutoDock

403 force field for small-molecule docking to zinc metalloproteins. Journal of chemical

404 information and modeling 54(8):2371-2379 DOI 10.1021/ci500209e.

405 Selvaraj S, Krishnaswamy S, Devashya V, Sethuraman S, Krishnan UM. 2014. Flavonoid-

406 metal ion complexes: a novel class of therapeutic agents. Medicinal Research Reviews

407 34(4):677-702 DOI 10.1002/med.21301.

408 Sherratt MJ, Hopkinson L, Naven M, Hibbert SA, Ozols M, Eckersley A, Newton VL, Bell

409 M, Meng QJ. 2019. Circadian rhythms in skin and other elastic tissues. Matrix Biology

$410 \quad$ 84:97-110 DOI 10.1016/j.matbio.2019.08.004.

411 Si YX, Yin S J, Oh S, Wang ZJ, Ye S, Yan L, Yang JM, Park YD, Lee J, Qian GY. 2012.

412 An integrated study of tyrosinase inhibition by rutin: progress using a computational

413 simulation. Journal of Biomolecular Structure and Dynamics 29(5):999-1012 DOI

$414 \quad 10.1080 / 073911012010525028$.

415 Szewczyk K, Miazga-Karska M, Pietrzak W, Komsta L, Krzemińska B, Grzywa-Celińska

416 A. 2020. Phenolic composition and skin-related properties of the aerial parts extract of

417 different Hemerocallis cultivars. Antioxidants 9(8):690 DOI 10.3390/antiox9080690.

418 Tanawattanasuntorn T, Thongpanchang T, Rungrotmongkol T, Hanpaibool C, Graidist P,

419 Tipmanee V. 2020. (-)-Kusunokinin as a potential aldose reductase inhibitor: equivalency

420 observed via AKR1B1 dynamics simulation. ACS Omega 6(1):606-614 DOI

$421 \quad$ 10.1021/acsomega.0c05102.

422 Teajaroen W, Phimwapi S, Daduang J, Klaynongsruang S, Tipmanee V, Daduang S. 2020.

423 A role of newly found auxiliary site in phospholipase A1 from Thai banded tiger wasp 
424 (Vespa affinis) in its enzymatic enhancement: in silico homology modeling and molecular 425 dynamics insights. Toxins 12(8):510 DOI 10.3390/toxins12080510.

426 Thring TS, Hili P, Naughton DP. 2009. Anti-collagenase, anti-elastase and anti-oxidant

427 activities of extracts from 21 plants. BMC complementary and alternative medicine 9(1):1-11

428 DOI $10.1186 / 1472-6882-9-27$

429 Widyowati R, Sugimoto S, Yamano Y, Sukardiman, Otsuka H, Matsunami K. 2016. New

430 isolinariins C, D and E, flavonoid glycosides from Linaria japonica. Chemical and

431 Pharmaceutical Bulletin 64(5):517-521 DOI 10.1248/cpb.c16-00073. 
Figure 1

Chemical structures of compounds.

(A), Bisresorcinol isolated from H. terminalis's trunk; (B), caffeic acid; (C), ursolic acid; and (D), $\beta$-arbutin.<smiles>CCC(=O)C=Cc1ccc(O)c(O)c1</smiles>

C

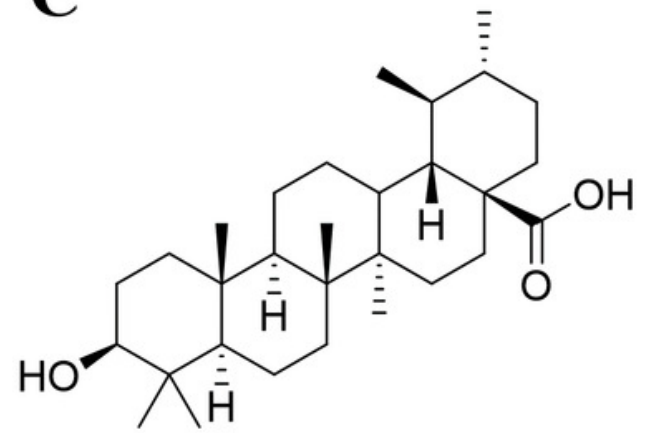

D<smiles>OCC1O[C@@H](Oc2ccc(O)cc2)C(O)C(O)C1O</smiles> 
Figure 2

Response of inhibition percentage

Response of \% inhibition, evaluated by linear regression analysis and correlation coefficients expressed as $\mathrm{R}^{2}$ shown in the panels; A, collagenase inhibition in compared to caffeic acid; $\mathrm{B}$, elastase inhibitory activity using ursolic acid as a positive control; and $C$, tyrosinase inhibitory assay in comparison with $\beta$-arbutin 
A



B

$\Delta$ Bisresorcinol $\quad$ Caffeic acid



$\Delta$ Bisresorcinol Ursolic acid

C



$\Delta$ Bisresorcinol $\mathbf{a} \beta$-arbutin 
Figure 3

Collagense bound ligands

(A) Molecular docking model of caffeic acid (red) and the bisresorcinol (blue) on the collagenase active site. Ligand interaction diagrams of caffeic acid (B-C) and the bisresorcinol (D-E), involving the $\pi-\pi$ stacking, hydrogen bond, and Van der Waals as respectively depicted in pink, green, and grey.

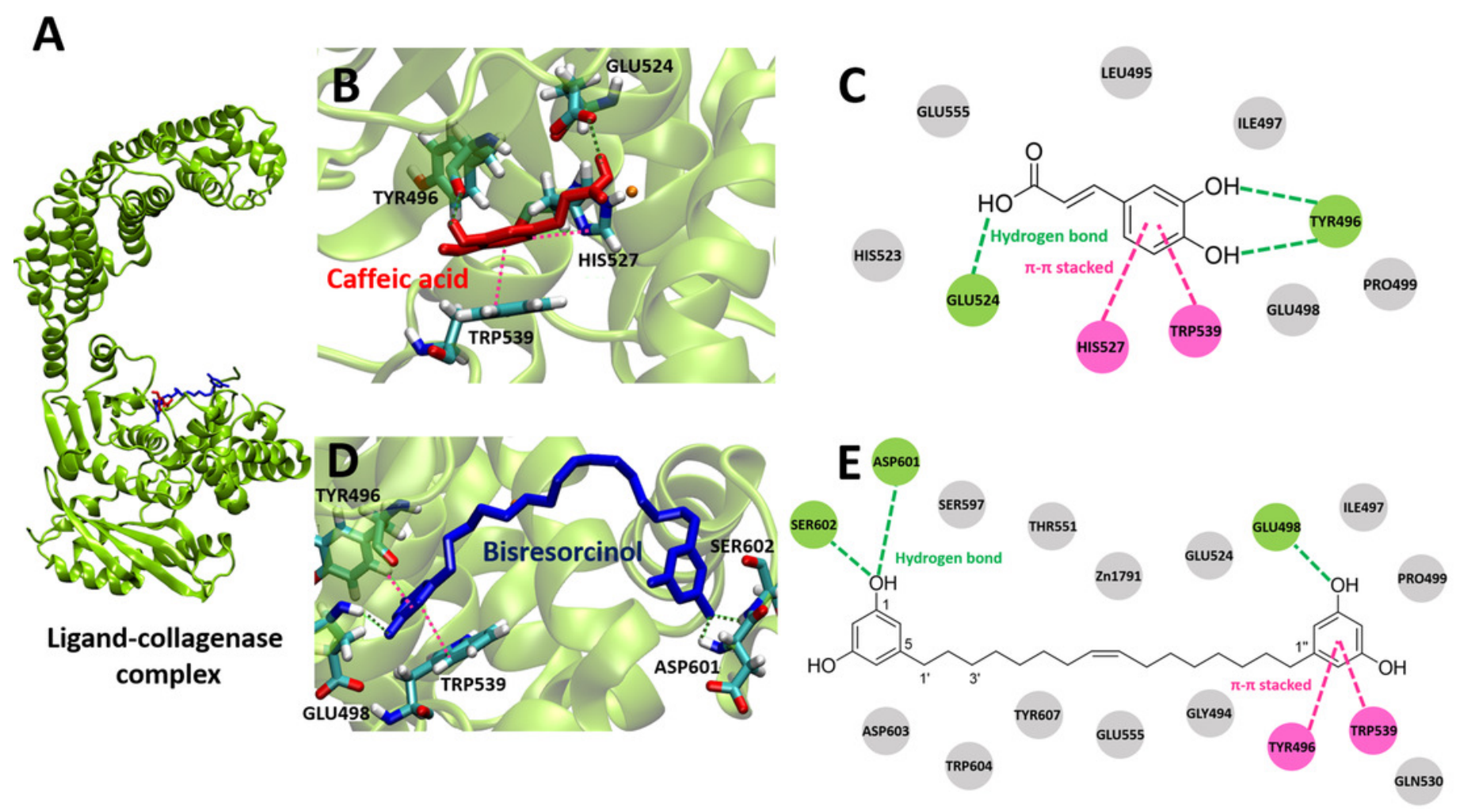


Figure 4

Elastase bound ligands

(A) Binding characteristics of the bisresorcinol (blue) and ursolic acid (red) to the active site of pig elastase; (B-C), Ligand-protein interaction diagrams of ursolic acid (B-C) and the bisresorcinol (D-E); Interactions involved $\pi-\pi$ stacking, hydrogen bonds, and Van der Waals force, depicted in pink, green, and grey, respectively.
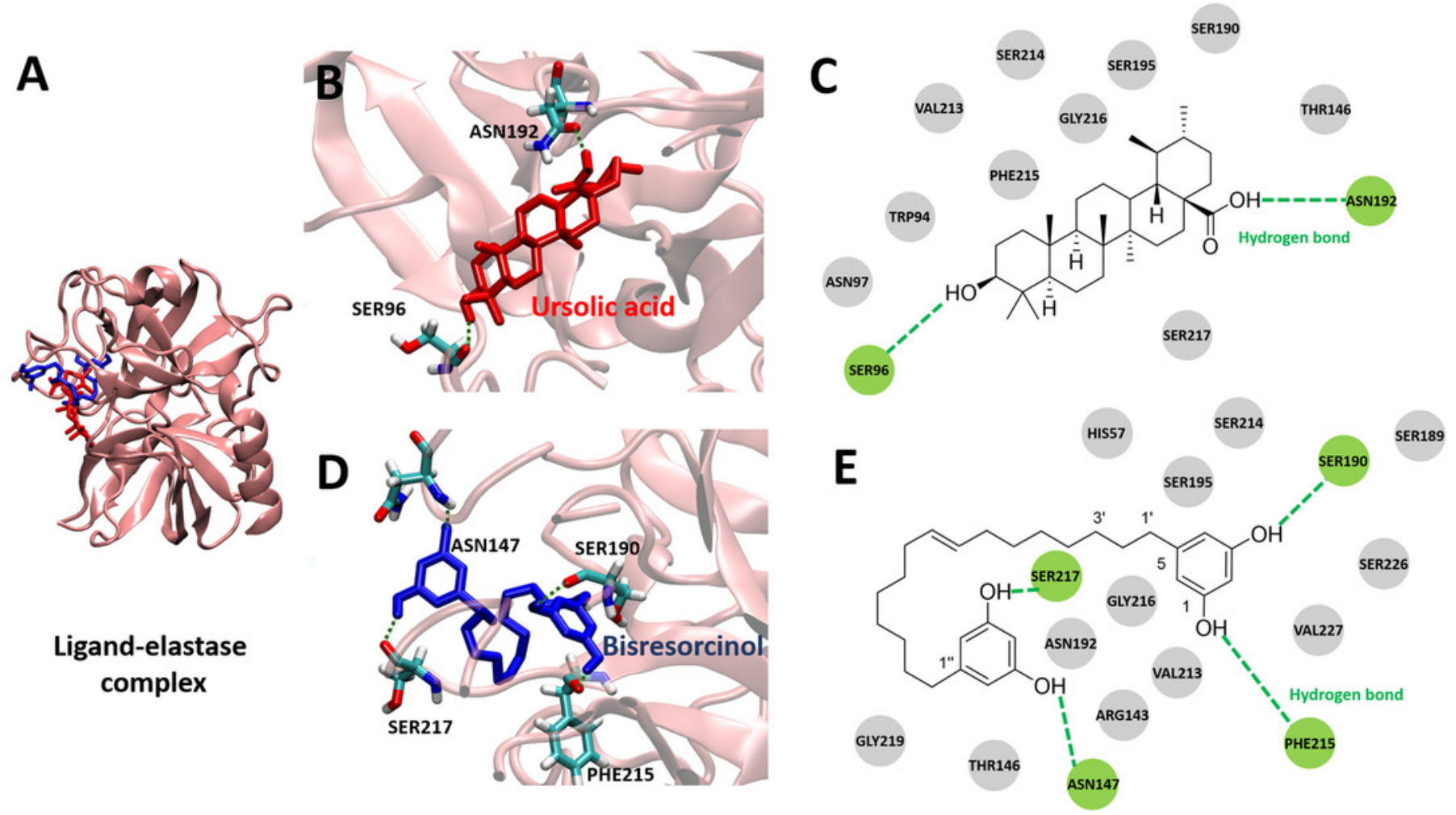
Figure 5

Tyrosinase bound ligands

(A) Molecular docking model of $\beta$-arbutin (red) and the bisresorcinol (blue) on the tyrosinase active site. Ligand-protein interaction diagrams of $\beta$-arbutin (B-C) and the bisresorcinol (D-E) showed the engagement of $\pi$-cation, $\pi-\pi$ stacking, hydrogen bonding, and van der waals force, depicted in orange, pink, green, and grey, respectively

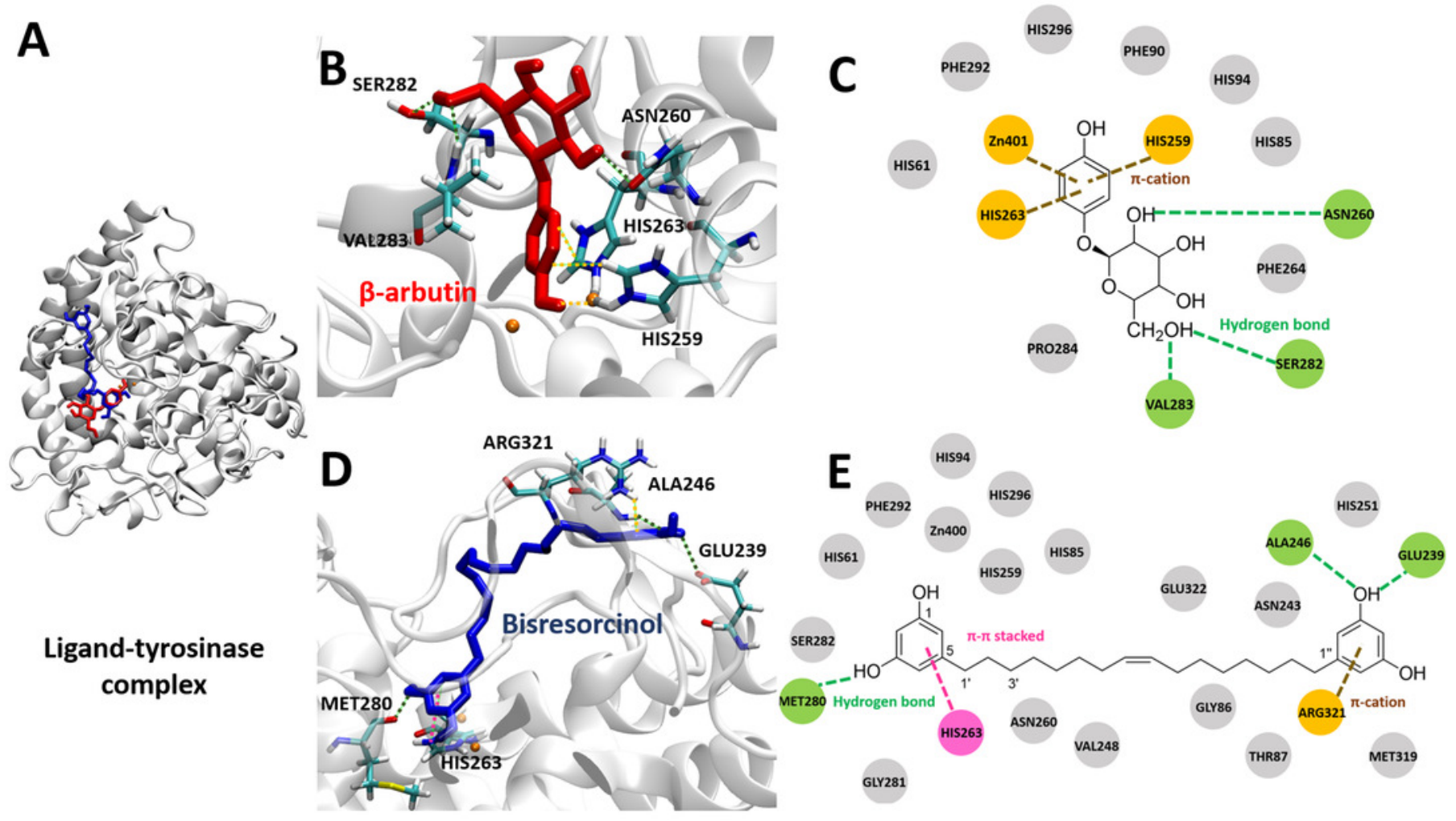


Figure 6

Binding modes of collagenase inhibitors

Binding modes of inhibitors namely caffeic acid, catechin, EGCG, and quercetin, and the bisresorcinol on collagenase enzyme; Symbols: red dash lines, hydrogen bonds; green dash lines, $\pi$-interactions.
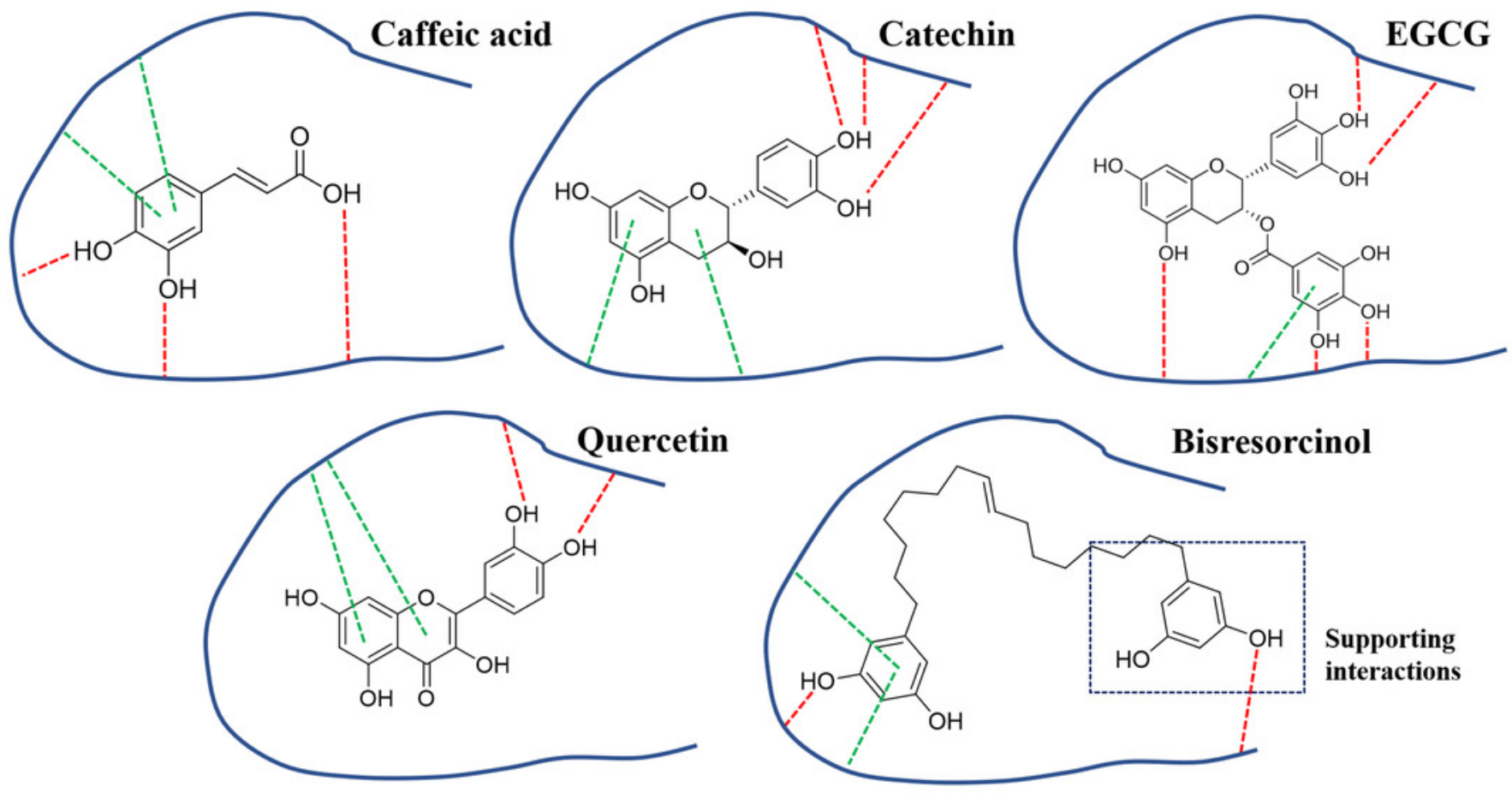
Figure 7

Binding modes of elastase inhibitors

Binding modes of actions of the bisresorcinol and known inhibitors, such as quercetin, procyanidin, and ursolic acid on elastase enzyme; Symbol: red dash lines, hydrogen bonds.

Figure 8

Binding modes of tyrosinase inhibitors

Binding modes of actions of the bisresorcinol and known inhibitors, e.g., kojic acid, Lmimosine, and rutin, on mushroom tyrosinase; Symbols: red dash lines, hydrogen bonds; green dash lines, $\pi$-interactions.



\section{Table $\mathbf{1}$ (on next page)}

The IC50 values of the bisresorcinal and positive standards for enzymatic inhibitory assays regarding collagenase, elastase, and tyrosinase; $*, p<0.01 ; * *, p<0.001$ 
1 Table 1 The $\mathrm{IC}_{50}$ values of the bisresorcinal and positive standards for enzymatic inhibitory

2 assays regarding collagenase, elastase, and tyrosinase; $*, p<0.01 ; * *, p<0.001$
Assay
$\mathrm{IC}_{50}\left(\mu \mathrm{mole} \mathrm{L}^{-1} \pm \mathrm{SEM}\right)$

\section{Collagenase Inhibition}

Bisresorcinol

Caffeic acid

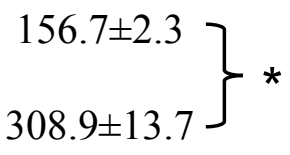

\section{Elastase Inhibition}

Bisresorcinal

$33.2 \pm 1.1$

Ursolic acid

$34.3 \pm 0.6$

\section{Tyrosinase Inhibition}

Bisresorcinol

$22.6 \pm 1.3$

$\beta$-Arbutin

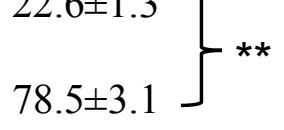

3 


\section{Table 2 (on next page)}

Table 2

Binding energy and amino acid residues that participated in the binding 
1 Table 2 Binding energy and amino acid residues that participated in the binding

\begin{tabular}{|c|c|c|}
\hline Ligand & $\begin{array}{c}\text { Binding energy } \\
\text { (kcal/mol) }\end{array}$ & Binding residue \\
\hline \multicolumn{3}{|c|}{ Collagenase inhibitor } \\
\hline Caffeic acid & -6.86 & $\begin{array}{l}\text { Glu524, Tyr496, } \\
\text { His527, Trp539, } \\
\text { Glu498, Glu555, His523, Ile497, } \\
\text { Leu495, Pro499 }\end{array}$ \\
\hline Bisresorcinol & -5.89 & $\begin{array}{l}\text { Asp601, Glu498, Ser602, } \\
\text { Tyr496, Trp539, } \\
\text { Asp603, Gln530, Glu524, Glu555, } \\
\text { Gly494, Ile497, Pro499, Thr551, } \\
\text { Trp604, Tyr607, Ser597, Zn1791 }\end{array}$ \\
\hline \multicolumn{3}{|l|}{ Elastase inhibitor } \\
\hline Ursolic acid & -8.94 & $\begin{array}{l}\text { Asn192, Ser96, } \\
\text { Asn97, Gly216, Phe215, Ser190, } \\
\text { Ser195, Ser214, Ser217, Thr146, Trp94, } \\
\text { Val213 }\end{array}$ \\
\hline Bisresorcinol & -5.69 & $\begin{array}{l}\text { Asn147, Phe215, Ser190, Ser217, } \\
\text { Asn192, Arg143, Gly216, Gly219, } \\
\text { His57, Ser189, Ser195, Ser214, Ser226 } \\
\text { Thr146, Val213, Val227 }\end{array}$ \\
\hline \multicolumn{3}{|c|}{ Tyrosinase inhibitor } \\
\hline$\beta$-arbutin & -5.52 & $\begin{array}{l}\text { His259, His263, Zn401 } \\
\text { Asn260, Ser282, Val283, } \\
\text { His61, His85, His94, His296, Phe90, } \\
\text { Phe264, Phe292, Pro284 }\end{array}$ \\
\hline Bisresorcinol & -6.57 & $\begin{array}{l}\text { Arg321, } \\
\text { Ala246, Glu239, Met280, } \\
\text { His263, } \\
\text { Asn243, Asn260, Glu322, Gly86, } \\
\text { Gly281, His61, His85, His } 94 \text {, His } 251 \text {, } \\
\text { His259, His296, Met319, Phe292, } \\
\text { Ser282, Thr87, Val248, Zn400 }\end{array}$ \\
\hline
\end{tabular}

Note: $\pi$-cation, $\pi-\pi$ stacking, and hydrogen bonds were depicted in italic, underline, and bold, respectively. The normal letter represented amino acids containing and Van der Waals interactions with the compound. 